\title{
$\operatorname{CON} F-951056--3$
}

UCRL-JC-121688

PREPRINT

\section{Rapid Mapping of Volumetric Errors}

\author{
D. Krulewich \\ L. Hale \\ D. Yordy.
}

This paper was prepared for submittal to the American Society for Precision Engineering Tenth Annual Meeting Austin, TX

October 15-20, 1995

September 13, 1995

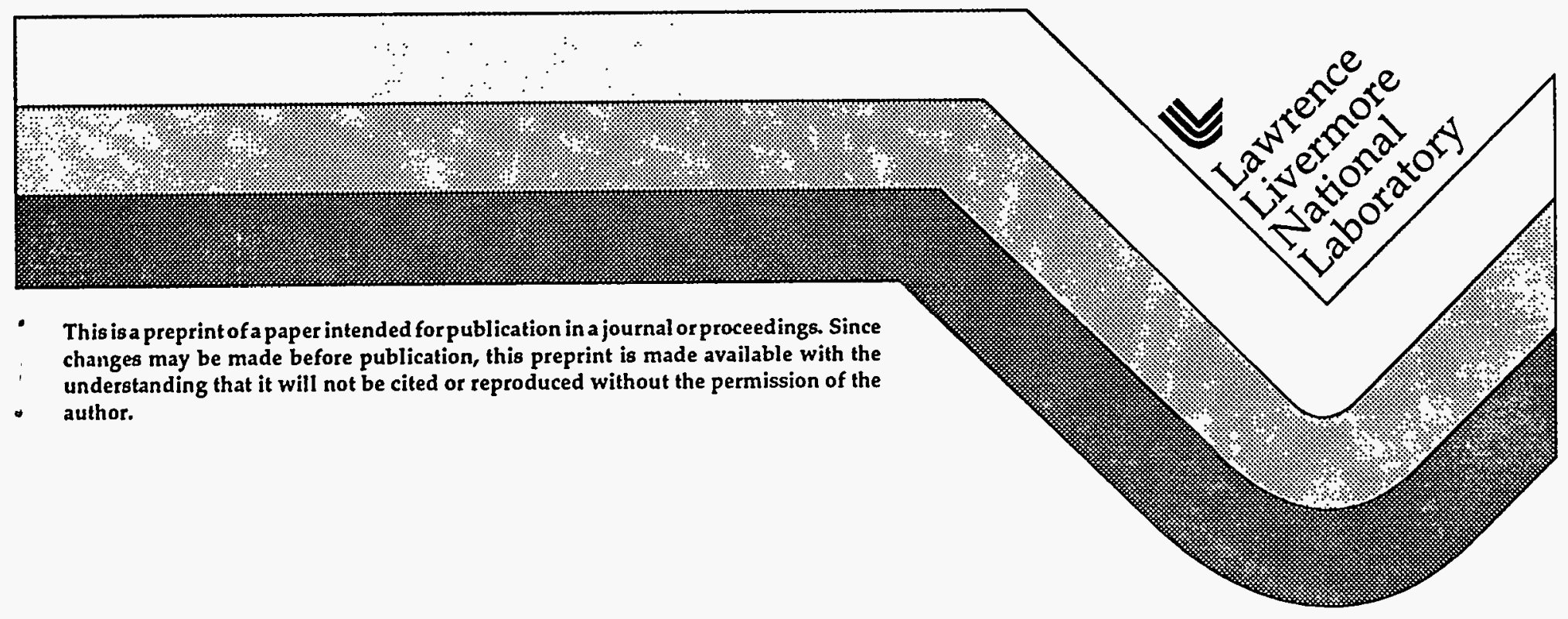




\section{DISCLAIMER}

This document was prepared as an account of work sponsored by an agency of the United States Government. Neither the United States Government nor the University of California nor any of their employees, makes any warranty, express or implied, or assumes any legal liability or responsibility for the accuracy, completeness, or usefulness of any information, apparatus, product, or process disclosed, or represents that its use would not infringe privately owned rights. Reference herein to any specific commercial product, process, or service by trade name, trademark, manufacturer, or otherwise, does not necessarily constitute or imply its endorsement, recommendation, or favoring by the United States Government or the University of California. The views and opinions of authors expressed herein do not necessarily state or reflect those of the United States Government or the University of California, and shall not be used for advertising or product endorsement purposes.

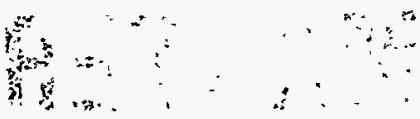




\title{
RAPID MAPPING OF VOLUMETRIC ERRORS
}

\author{
Debbie Krulewich, Layton Hale, Don Yordy \\ Lawrence Livermore National Laboratory \\ Livermore, CA 94550
}

\section{Abstract}

This paper describes a relatively inexpensive, fast, and easy to execute approach to mapping the volumetric errors of a machine tool, coordinate measuring machine, or robot. An error map is used to characterize a machine or to improve its accuracy by compensating for the systematic errors. The method consists of three steps: (1) modeling the relationship between the volumetric error and the current state of the machine; (2) acquiring error data based on length measurements throughout the work volume; and (3) optimizing the model to the particular machine.

\section{Background}

There are three documented methods for modeling volumetric errors: (1) volumetric error modeling, (2) parametric error modeling, and (3) functional error modeling. Volumetric error modeling involves measureing the volumetric errors throughout the working space of the machine. The error at a given location is determined by interpolation between the measured points. For good accuracy, many points must be measured. Furthermore, to date an adequate measuring device does not exist. Parametric error modeling, the most commonly used method, measures the six parametric errors of each moving element and then combines them based on the geometry of the machine to form a functional point to workpiece error. Parametric error modeling is often not practical due to the complexity, time requirements, and expense. Functional error modeling represents the volumetric errors as a function of the current state of the machine (commanded position, temperature, etc.), and the function is fit to volumetric error measurements. Because the model provides an intelligent way to interpolate between measured points, fewer measurements are required. Current technologies for acquiring volumetric error measurements include probing a known artifact, but it is difficult to acquire an adequate density of measurement points. Alternatively, we are using a new measurement technique based on length measurements for acquiring volumetric error measurements.

\section{Model Development}

The functional model is built from the parametric error model. The parametric errors are appropriately combined to predict the error between the functional point and workpiece. Mathematical expressions for each parametric error component are then substituted into the parametric error model. As a first attempt, we have used simple polynomials that are only dependent on commanded position. If no nonrigid coupling between axes is expected, the mathematical expression for each parametric error will only be dependent on the moving axis position; however, if non-rigid coupling is expected, the mathematical expression may contain other axes. It should be noted that the model development requires full knowledge of the machine behavior for a given machine design. However, the model is characteristic of all machines with that design so that the general model only needs to be developed once.

In the future, we will investigate other functional forms such as orthogonal polynomials and splines. We also plan to incorporate quasi-static thermal and load induced errors into the model. Along with the current location of the machine, the model would then include current temperatures of critical locations on the machine and the part weight. 


\section{Measurement Technique}

A series of length measurements, taken from several fixed base locations to various functional points in the work volume, are compared with the respective lengths calculated between the commanded positions and the nominal base locations. The difference between measured and calculated lengths to a point is approximately equal to the component of error projected onto that measurement direction. This difference is then set equal to the projected error, expressed as a volumetric function. The parameters of the function are solved to optimize its global fit to all the measurements.

The notation in Table 1 will be used throughout. The error vector is defined as

$$
e=L_{m}-L_{c} \text {. }
$$

and the projection of the error vector onto the nominal commanded length vector is

$\frac{\mathbf{L}_{\mathrm{c}}}{L_{c}} \bullet \mathbf{e}=\frac{\mathbf{L}_{\mathrm{c}}}{L_{c}} \cdot\left(\mathbf{L}_{\mathbf{m}}-\mathbf{L}_{\mathbf{c}}\right)=\frac{\mathbf{L}_{\mathbf{c}}}{L_{c}} \cdot \mathbf{L}_{\mathbf{m}}-L_{c}$.

Because the error is small with respect to the measured length, the projection of the measured length vector onto the commanded length vector is approximately the magnitude of the measured length,

$$
\frac{\mathbf{L}_{\mathrm{c}}}{L_{c}} \cdot \mathrm{L}_{\mathrm{m}} \approx L_{m} \text {. }
$$

Equation 2 then reduces to

$$
d L \equiv \frac{\mathbf{L}_{\mathrm{c}}}{L_{c}} \bullet \mathrm{e} \approx L_{m}-L_{c} .
$$
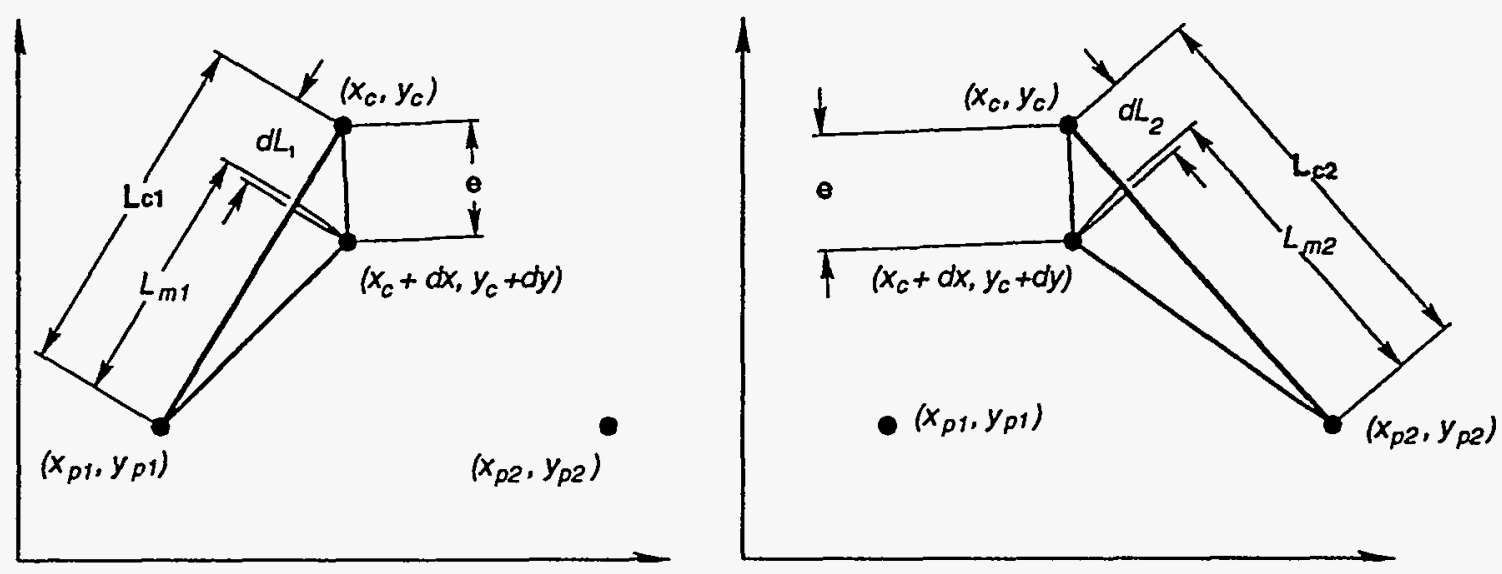

Table 1: Notations

\begin{tabular}{|l|l|}
\hline$\left(x_{c}, y_{c}, z_{c}\right)$ & commanded machine position \\
$\left(x_{p}, y_{p}, z_{p}\right)$ & nominal base location \\
$\mathbf{e}$ & error vector, $(d x, d y, d z)$ \\
$\mathbf{L}_{\mathbf{c}}$ & nominal commanded length \\
& vector, $\left(x_{c}, y_{c}, z_{c}\right)-\left(x_{p}, y_{p}, z_{p}\right)$ \\
$L_{c}$ & $\begin{array}{l}\text { magnitude of the nominal } \\
\text { commanded length }\end{array}$ \\
& $\begin{array}{l}\text { measured length direction } \\
\mathbf{L}_{\mathbf{m}}\end{array}$ \\
$L_{\mathrm{m}}$ & (unknown) \\
$d L$ & measured length \\
projection of e onto $\mathbf{L}_{c}$
\end{tabular}

Figure 1 illustrates the error projections in a two-dimensional plane. In this twodimensional example, two lengths, $L_{m l}$ and $L_{m 2}$ are measured from two nominal base locations, $\left(x_{p 1}, y_{p 1}\right)$ and $\left(x_{p 2}, y_{p 2}\right)$, to the functional point located at $\left(x_{c}, y_{c}\right)+(d x, d y)$. The error vector e, projected onto the commanded vectors, $\mathbf{L}_{\mathbf{c l}}$ and $\mathbf{L}_{c 2}$, yields lengths, $d L_{1}$ and $d L_{2}$, that are approximately equal to the respective differences between the commanded and measured lengths.

Figure 1: Simple 2-D Representation of Error Projections 
The same reasoning applies to three dimensions, so that Equation 4 is completely general. Expanding Equation 4 for the threedimensional case results in

$$
\frac{\left(x_{c}-x_{p}\right)}{L_{c}} d x+\frac{\left(y_{c}-y_{p}\right)}{L_{c}} d y+\frac{\left(z_{c}-z_{p}\right)}{L_{c}} d z=L_{m}-L_{c} \text {. }
$$

To solve for the three unknowns, $d x, d y$, and $d z$, in Equation 5, lengths from at least three base locations must be measured. Applying Equation 5 to only three measurements, one from each base location to a single point, would result in three linear equations that can be solved directly for the three-dimensional volumetric error. This is the linearized form of trilateration. This method of projecting errors onto the nominal commanded length vector, which we refer to as the error projection method, is naturally extendible to any number of measurements for the purpose of fitting the functional model. This is accomplished by substituting models for $d x$, $d y$, and $d z$ into Equation 5. Then lengths from three or more base locations to the functional point are measured for commanded positions throughout the working volume. Applying Equation 5 to each commanded position results in an overdetermined set of equations that can be solved for the unknown model parameters using linear regression.

The non-systematic errors tend to be smoothed during the fitting procedure. If (1) the error model is correct and (2) the non-systematic errors appear to be random, the model fitting will have an averaging effect on the errors. To ensure that time dependent errors appear to be random, the measurement points are taken in a random order to disassociate time and position. For example, the measuring device, if accidentally warmed by the operator, will slowly cool causing measurement errors that are associated with time. If the measurements were taken in a particular order, for instance always increasing in the $x$ direction, then the time related errors will couple to the $x$-axis motion. Unavoidable time dependent errors can be reduced by randomizing the order of measurements so their random appearance will average in the fitting process.

\section{Model Optimization}

To ensure that the error model is correct, the general model for a given machine design must be optimized for the specific machine. The general model that describes all for a given design will have more terms than required for most machines. The extra terms hurt the predictive ability of the model because the extra terms begin to fit the noise in the data. Therefore, the best subset model must be selected for each particular machine. Unless the model is orthogonal, every possible subset must be considered. To find the best model, I have had most success using Mallows $C_{p}$ statistic, which is an unbiased estimator of the total sum of squares of discrepancies (variance error plus bias error) of the candidate model from the true but unknown model, and is calculated by

where

$$
C_{p}=\frac{R S S_{p}}{s^{2}}-(n-2 p)
$$

$$
\begin{aligned}
R S S_{p} & =\text { residual sum squared error from } \\
& \text { a candidate model containing } p \\
& \text { parameters, } \\
& =\text { number of the parameters in the } \\
s^{2}= & \text { candidate model, } \\
& \text { from the general model. } \\
& \text { Since the expected value of } C_{p} \text { is } p,
\end{aligned}
$$
adequate models will show up as points close to $C_{p}=p$. Using actual data, I have selected the best subset model and found that the optimized model fit an independently acquired data set better than the full model.

\section{Results}

The volumetric error map represents the systematic portion of the error, where the apparently non-systematic errors are those that are not taken into account in the model. The degree of systematic error that can accurately be is controlled by the number of measurements and the non-systematic errors of the length measuring device and the machine. During initial testing, we acquired 1500 length measurements throughout the volume of a three-axis machine in three hours using a Laser 
Ball Bar manufactured by Tetra Precision. Model predictions were then compared to independently acquired body and face diagonal errors measured with a laser interferometer. The model predictions were within $2 \mu \mathrm{m}$ of the diagonal measurements, which is the combined non-systematic error of the measuring device and the machine. Given an infinite amount of data, the prediction should exactly predict the apparently systematic portion of the machine error assuming that the model is appropriate and that the measurements are taken throughout the volume.

\section{Conclusion}

In conclusion, the functional error model can accurately represent the volumetric errors throughout the working space of a machine. Furthermore, the error projection method provides a means to recover volumetric errors from rapidly acquired length measurements. The reduction in time required to map the volume is the benefit of this method rather than improved accuracy over current technologies. However, the accuracy of the error map is comparable to that achieved using conventional methods. We have not explored the use of this method on ultra-precision machine tools and coordinate measuring machines. A length measuring device with adequate accuracy would be required.
1. Belforte, G.; Bona, B.; Canuto, F.; Donati, F.; Ferraris, F.; Gorini, I.; Morei, S.; Peisino, M.; Sartori, S. Coordinate Measuring Machines and Machine Tools Selfcalibration and Error Correction. Annals of the CIRP. 1987; Vol. 36(No. 1): pp. 359-364.

2. Domnez, M.; Blomquist, R.; Hocken, R.; Liu, C.; Barash, M. General Methodology for Machine Tool Accuracy Enhancement by Error Compensation. Precision Engineering. 1986 Oct; Vol. 8(No. 4): pp. 187-196.

3. Furnival, G.M. (Yale University).Regressions by Leaps and Bounds. Technometrics. November 1974; Vol. 16(No. 4): pp. 499-511.

4. Kiridena, V. S. B.; Ferreira, P. M. (University of IL). Parameter Estimation and Model Verification of First Order Quasistatic Error Model ${ }^{\circ}$ for Three-Axis Machining Centers. International Journal of Machine Tools and Manufacturing. 1994; Vol. 34(No. 1): pp. 101-125.

5. Mallows, C.L. (Bell Laboratories). Some Comments on $\mathrm{Cp}$. Technometrics. November 1973; Vol. 15(No. 4): pp. 661675.

6. Soons, J. A.; Schellekens, P. H. (Eindhoven University of Technology). On the Calibration of Multi-Axis Machines Using Distance Measurements. Proc. ISMQC. 1992: pp. 321-340.

7. Soons, J.; Theuws, F.; Schellekens, P. (Eindhoven University of Technology). Modeling the Errors of Multi-Axis Machines: A General Methodology.
Precision Engineering. 1992 Jan; Vol. 14(No. 1): pp. 5-19.

8. Teeuwsen, J.; Soons, J.; Schellekens, P. (Eindhoven University of Technology, Netherlands). A General Method for Error Description of CMM's Using Polynomial Fitting Procedures. Annals of the CIRP. 1989; Vol. 38(No. 1): pp. 505-510.

9. Zhang, G.; Chu, H.; Tang, W.; Jin, Z. (Tianjin University). Distance-Distance Method for Straightness Measurement. Annals of the CIRP. 1992; Vol. 41(No. 1): pp. 581-584.

10. Zhang, G.; Ouyang, R.; Lu, B.; Hocken, R. A Displacement Method for Machine Geometry Calibration. Annals of the CIRP. 1988; Vol. 37(No. 1): pp. 515-518.

11. Ziegert, J. C.; Mize, C. D. (Department of Mechanical Engineering, University of Florida). The Laser Ball Bar: A New instrument for Machine Tool Metrology. Journal of the American Society for Precision Engineering. 1994 Oct; Vol. 16(No. 4): pp. 259-267. 



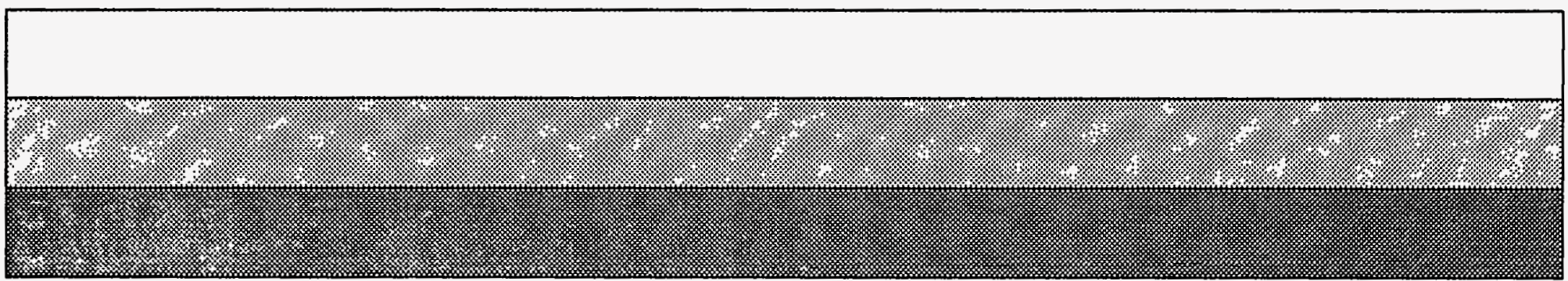

